\title{
Aggregation Operators and Quadric Hypersurfaces
}

\author{
J.Recasens
}

\begin{abstract}
Aggregation operators that are quadric hypersurfaces are studied. The interest lays in the fact that the most popular aggregation operators are indeed quadric hypersurfaces. Keywords: Aggregation Operator, Idempotent, Symmetric, Quadric Hypersurface.
\end{abstract}

\section{Introduction}

If some laymen were asked to aggregate two or more numerical values, they would probably suggest the use of the arithmetic mean. More sophisticated people would suggest the geometric, quadratic or harmonic mean while experts would also propose the use of the maximum t-conorm, the minimum t-norm, Łukasiewicz and product t-norms and t-conorms or OWA operators. These are indeed the most popular aggregation operators and they are (part of) ruled quadratic hyper surfaces. Apart form the Eukasiewicz and product t-norms and t-conorms, they are idempotent as well.

This paper studies other ruled quadric surfaces that correspond to aggregation operators in two variables. In this way new families of aggregation operators, some of them combinations of the previous ones, are obtained. The results are then generalized to aggregation operators in several variables that are (part of) quadric hypersurfaces.

Let us recall the definition of aggregation operator.

\footnotetext{
J. Recasens

Sec Matemàtiques i Informàtica

ETS Arquitectura del Vallès

Universitat Politècnica de Catalunya

C. Pere Serra 1-15

08190 Sant Cugat del Vallès

Spain e-mail: j.recasens@upc.edu
} 
Definition 1. [2] An aggregation operator is a map $h: \bigcup_{n \in N}[0,1]^{n} \rightarrow[0,1]$ satisfying

1. $h(0, \ldots, 0)=0$ and $h(1, \ldots, 1)=1$

2. $h(x)=x \forall x \in[0,1]$

3. $h\left(x_{1}, \ldots, x_{n}\right) \leq h\left(y_{1}, \ldots, y_{n}\right)$ if $x_{1} \leq y_{1}, \ldots, x_{n} \leq y_{n}$ (monotonicity).

$h$ is idempotent if and only if $h(\overbrace{x, \ldots, x}^{n \text { times }})=x$ for all $x \in[0,1]$ and for all $n \in N$.

$h$ is symmetric if and only if $h\left(x_{1}, \ldots, x_{n}\right)=h\left(x_{\pi(1)}, \ldots, x_{\pi(n)}\right)$ for any permutation $\pi$ of $\{1,2, \ldots, n\}$.

Given $n \in N$, the restriction of an aggregation operator $h$ to $[0,1]^{n}$ will be called an aggregation operator in $n$ variables.

\section{Ruled Quadric Surfaces}

In this section the idempotent ans symmetric aggregation operators in two variables that are quadric surfaces will be studied.

Definition 2. A quadric surface is a surface defined in implicit form by a second degree polynomial

$$
a x^{2}+b y^{2}+c z^{2}+d x y+e x z+f y z+g x+h y+i z+j=0 .
$$

In order to find the ruled quadric surfaces which are aggregation operators, we will consider separately the cases $c \neq 0$ and $c=0$.

\subsection{Case $c=0$}

If $c=0$, then isolating $z$ from (1) we obtain

$$
z=-\frac{a x^{2}+b y^{2}+d x y+g x+h y+j}{e x+f y+i} .
$$

Replacing $e$ by $-e, f$ by $-f$ and $i$ by $-i,(2)$ is

$$
z=\frac{a x^{2}+b y^{2}+d x y+g x+h y+j}{e x+f y+i} .
$$

If we want the last map to be symmetric, we must have $b=a, g=h$ and $e=f$, obtaining

$$
z=\frac{a x^{2}+a y^{2}+d x y+g x+g y+j}{e x+e y+i} .
$$


$z(0,0)$ must be 0 . From this we have $j=0$.

If $z$ is idempotent, writing explicitly $z(x, x)=x$ we obtain

$$
z(x, x)=\frac{2 a x^{2}+d x^{2}+2 g x}{2 e x+i}=x
$$

or

$$
(2 a-2 e+d) x^{2}=(i-2 g) x .
$$

This equation is satisfied for all $x \in[0,1]$ if and only if

$$
d=2 e-2 a \text {. }
$$

and

$$
i=2 g \text {. }
$$

The formula of the quadric surface becomes then

$$
z=\frac{a x^{2}+a y^{2}+(2 e-2 a) x y+g x+g y}{e x+e y+2 g} .
$$

Now we can consider two cases: $e \neq 0$ and $e=0$.

\subsubsection{Case $c=0$ and $e \neq 0$}

In this case we can divide the numerator and the denominator of (3) by $e$. Renaming $\frac{a}{e}$ by $a$, and $\frac{g}{e}$ by $g$, we get

$$
z=\frac{a(x-y)^{2}+2 x y+g x+g y}{x+y+2 g} .
$$

The denominator must be different from 0 for all $x, y \in(0,1)$. This means

$$
g \geq 0 \text { or } g \leq-1
$$

For $x=0$ and $y=1$, we obtain

$$
z(0,1)=\frac{a+g}{1+2 g}
$$

This value must be between 0 and 1 . Imposing that it must be greater or equal than 0 , we obtain the following conditions for $a$ and $g$.

$$
g \geq-\frac{1}{2} \text { and } a \geq-g
$$

or

$$
g \leq-\frac{1}{2} \text { and } a \leq-g
$$


Imposing that it must be smaller or equal than 1, we obtain the following conditions for $a$ and $g$.

$$
g \geq-\frac{1}{2} \text { and } a \leq g+1
$$

or

$$
g \leq-\frac{1}{2} \text { and } a \geq g+1
$$

The partial derivatives $\frac{\partial z}{\partial x}(1,0)$ and $\frac{\partial z}{\partial x}(0,1)$ must be greater or equal than 0 .

$$
\frac{\partial z}{\partial x}(1,0)=\frac{(2 a+g)(1+2 g)-a-g}{(1+2 g)^{2}} \geq 0
$$

is satisfied if and only if

$$
g \geq-\frac{1}{4} \text { and } a \geq \frac{-2 g^{2}}{1+4 g}
$$

or

$$
\begin{gathered}
g \leq-\frac{1}{4} \text { and } a \leq \frac{-2 g^{2}}{1+4 g} . \\
\frac{\partial z}{\partial x}(0,1)=\frac{(-2 a+g+2)(1+2 g)-a-g}{(1+2 g)^{2}} \geq 0
\end{gathered}
$$

is satisfied if and only if

$$
g \geq-\frac{3}{4} \text { and } a \leq \frac{2+2 g^{2}+4 g}{3+4 g}
$$

or

$$
g \leq-\frac{3}{4} \text { and } a \geq \frac{2+2 g^{2}+4 g}{3+4 g} .
$$

Summarizing, the conditions on $g$ and $a$ are

$$
g \geq 0 \text { and } \frac{-2 g^{2}}{1+4 g} \leq a \leq \frac{2+2 g^{2}+4 g}{3+4 g}
$$

or

$$
g \leq-1 \text { and } \frac{2+2 g^{2}+4 g}{3+4 g} \leq a \leq \frac{-2 g^{2}}{1+4 g} .
$$

\subsubsection{Case $c=0$ and $e=0$}

In this case, putting $\frac{a}{2 g}=b$,

$$
z=b(x-y)^{2}+\frac{x+y}{2} .
$$

$z(1,0)$ is then 


$$
b+\frac{1}{2}
$$

Imposing again that this value must be between 0 and 1 , we get that

$$
-\frac{1}{2} \leq b \leq \frac{1}{2}
$$

Imposing that the partial derivative $\frac{\partial z}{\partial x}(1,0)$ must be greater or equal than 0 , we get

$$
b \geq-\frac{1}{4}
$$

Imposing that the partial derivative $\frac{\partial z}{\partial x}(0,1)$ must be greater or equal than 0 , we get

$$
b \leq \frac{1}{4} \text {. }
$$

Summarizing,

$$
-\frac{1}{4} \leq b \leq \frac{1}{4}
$$

2.2 Case $c \neq 0$

If $c \neq 0$, we can divide (1) by $c$. Renaming $\frac{a}{c}$ by $-a, \frac{b}{c}$ by $-b$, etc, we obtain

$$
\begin{gathered}
z=\frac{1}{2}((e x+f y+i \pm \\
\left.\sqrt{(e x+f y+i)^{2}-4 a x^{2}-4 b y^{2}-4 g x-4 d x y-4 h y-4 j}\right) .
\end{gathered}
$$

If we impose symmetry we get

$$
\begin{gathered}
z=\frac{1}{2}(e x+e y+i \pm \\
\left.\sqrt{(e(x+y)+i)^{2}-4 a x^{2}-4 a y^{2}-4 g x-4 d x y-4 g y-4 j}\right) .
\end{gathered}
$$

We can distinguish the cases where the square root is added or subtracted.

\subsubsection{Adding the square root}

In this case, (4) becomes

$$
\begin{gathered}
z=\frac{1}{2}(e x+e y+i+ \\
\left.\sqrt{(e(x+y)+i)^{2}-4 a x^{2}-4 a y^{2}-4 g x-4 d x y-4 g y-4 j}\right) .
\end{gathered}
$$

Imposing $z(0,0)=0$, we get

$$
i+\sqrt{i^{2}-4 j}=0
$$

and therefore $i \leq 0$ and $j=0$. 
From $z(1,1)=1$, we get

$$
2=2 e+i+\sqrt{(2 e+i)^{2}-8 a-8 g-4 d}
$$

and form this, $1-2 e-i+2 a+2 g+d=0$.

From $z\left(\frac{1}{2}, \frac{1}{2}\right)=\frac{1}{2}$, we get

$$
1=e+i+\sqrt{(e+i)^{2}-2 a-4 g-2 d}
$$

and form this, $1-2 e-2 i+2 a+4 g+d=0$.

So $i=2 g$ (and $g \leq 0$ ) and $d=-1+2 e-2 a$.

Now imposing $z(k, k)=k$ we get

$$
k=\frac{1}{2}\left(2 e k+i+\sqrt{(2 k e+i)^{2}-8 a k^{2}-8 g k-4 d k^{2}}\right) .
$$

which is equivalent to

$$
\begin{gathered}
k=\frac{1}{2}\left(2 e k+i+\sqrt{(2 k e+i)^{2}-8 e k^{2}-4 i k+4 k^{2}}\right)= \\
\frac{1}{2}\left(2 e k+i+\sqrt{(2 k e+i-2 k)^{2}}\right) .
\end{gathered}
$$

Then

$$
2 k e+i \leq 2 k \text { for all } k \in[0,1] .
$$

This is satisfied for all $k \in[0,1]$ if and only if

$$
2 e+i \leq 2 .
$$

Putting $b=\frac{e}{2}$, the equation of the quadric surface is then

$$
\begin{gathered}
z=b(x+y)+g+ \\
\sqrt{(b(x+y)+g)^{2}-a(x-y)^{2}-g(x+y)+(1-4 b) x y} .
\end{gathered}
$$

and (5) becomes

$$
\begin{gathered}
2 b+g \leq 1 . \\
z(1,0)=b+g+\sqrt{b+g)^{2}-a-g}
\end{gathered}
$$

which implies

$0 \leq z(1,0) \leq 1$ gives

$$
g+a \leq(b+g)^{2} .
$$

and if $b+g \leq 0$, then

$$
b+g \leq 1, g+2 b-a \leq 1
$$

Now imposing that $\frac{\partial z}{\partial x}(1,0) \geq 0$ we obtain

$$
a+g \leq 0 .
$$

$$
b+\frac{1}{2} \frac{2 b^{2}+2 b g-2 a-g}{\sqrt{(b+g)^{2}-a-g}} \geq 0
$$

and $\frac{\partial z}{\partial x}(0,1) \geq 0$ gives 


$$
b+\frac{1}{2} \frac{2 b^{2}+2 b g+2 a-g+1-4 b}{\sqrt{(b+g)^{2}-a-g}} \geq 0 .
$$

\subsubsection{Subtracting the square root}

Similar calculations as in the previous subsection leads to

$$
\frac{z=b(x+y)+g-}{\sqrt{(b(x+y)+g)^{2}-a(x-y)^{2}-g(x+y)+(1-4 b) x y}}
$$

with

$$
\begin{aligned}
& g \geq \max (0,1-2 b,-b) \\
& a \leq \min \left((b+g)^{2}-g, 1+g+2 b\right) \\
& b-\frac{1}{2} \frac{2 b^{2}+2 b g-2 a-g}{\sqrt{(b+g)^{2}-a-g}} \geq 0 \\
& b-\frac{1}{2} \frac{2 b^{2}+2 b g+2 a-g+1-4 b}{\sqrt{(b+g)^{2}-a-g}} \geq 0 \\
& \text { If } b+g \geq 1, \text { then } g+2 b-a \geq 1 .
\end{aligned}
$$

\section{Summarizing the results for two variables}

Table 1 summarizes the results obtained in the previous section.

- If in the first equation $a=g=0$, we recover the harmonic mean.

- If in the second equation $b=0$, we recover the arithmetic mean.

- If in the third equation $b=g=a=0$ we recover the geometric mean.

- If in the third equation $a=-\frac{1}{2}$ and $b=g=0$, we recover the quadratic mean.

- If in the third equation $a=g=0$ and $b=\frac{1}{2}$, we recover the Maximum aggregation operator.

- If in the fourth equation $a=g=0$ and $b=\frac{1}{2}$, we recover the Minimum aggregation operator.

- If $\frac{1}{2} \leq p \leq 1$ and in the third equation $a=g=0$ and $b=\frac{p}{2}$, we recover the OWA operator with weights $p$ and $1-p$.

- If $0 \leq p \leq \frac{1}{2}$ and in the fourth equation $a=g=0$ and $b=\frac{p}{2}$, we recover the OWA operator with weights $p$ and $1-p$.

Table 2 shows the ruled quadric surfaces corresponding to Table 1.

\section{Aggregation operators in more than two variables}

Aggregation operators in $n$ variables corresponding to quadric hypersurfaces can be studied in a similar way. In this case, the equation of the corresponding quadric hypersurface is

$$
\sum_{i=0}^{n} a_{i} x_{i}^{2}+\sum_{i, j=0, i>j}^{n} b_{i j} x_{i} x_{j}+\sum_{i=0}^{n} c_{i} x_{i}+d=0
$$

and we want to isolate a variable (say $x_{0}, x_{0}=f\left(x_{1}, x_{2}, \ldots, x_{n}\right)$ ). 
Table 1 Ruled quadric surfaces that are idempotent and symmetric aggregation operators

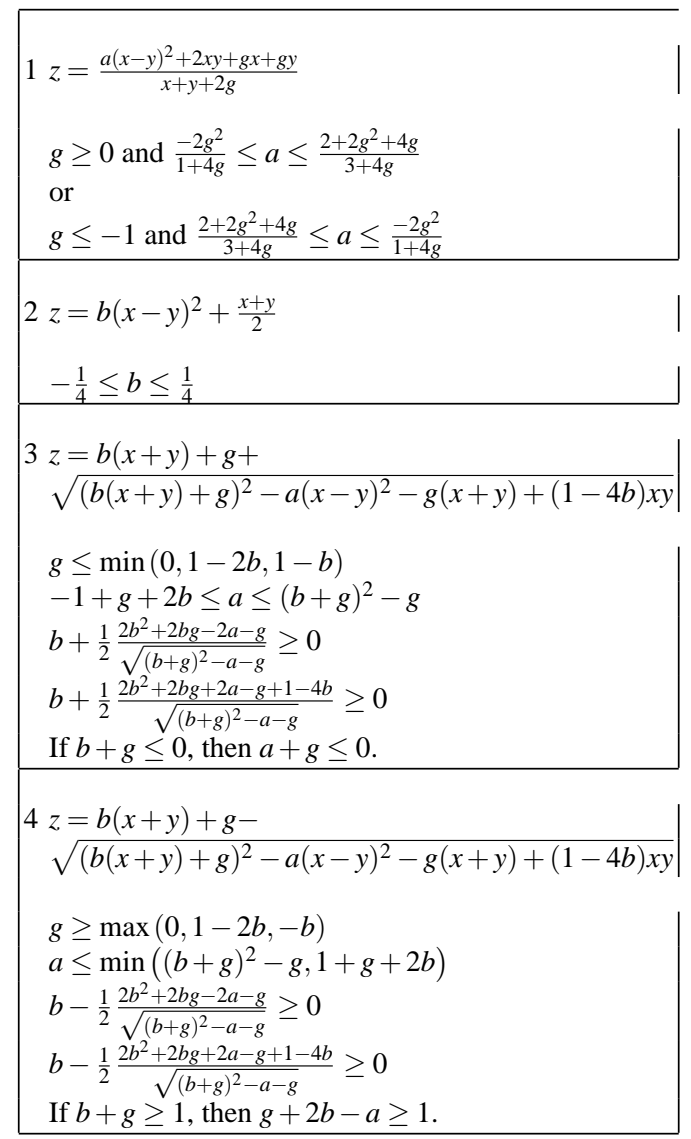

1. If $a_{0}=0$, then

$$
x_{0}=-\frac{\sum_{i=1}^{n} a_{i} x_{i}^{2}+\sum_{i, j=1, i>j}^{n} b_{i j} x_{i} x_{j}+\sum_{i=1}^{n} c_{i} x_{i}+d}{\sum_{i=1}^{n} b_{0 i} x_{i}+c_{0}}
$$

Imposing symmetry and $f(0,0, \ldots, 0)=0$ we get

$$
x_{0}=\frac{a \sum_{i=1}^{n} x_{i}^{2}+b \sum_{i, j=1, i>j}^{n} x_{i} x_{j}+c \sum_{i=1}^{n} x_{i}}{d \sum_{i=1}^{n} x_{i}+e}
$$

Idempotency leads to

$$
b=\frac{2(d-a)}{n-1}
$$

and

$$
e=n c \text {. }
$$

The quadric hypersurface is then 


\section{Table 2}

$$
\begin{aligned}
& \begin{array}{|l|}
\hline 1 \quad z=\frac{a(x-y)^{2}+2 x y+g x+g y}{x+y+2 g} \\
\\
\text { If } a \neq \frac{1}{2} \text {, then it is a cone } \\
\text { If } a=\frac{1}{2} \text {, then it is a couple of non-parallel planes } \\
\hline 2=b(x-y)^{2}+\frac{x+y}{2} \\
\text { If } b \neq \frac{1}{4}, \text { then it is a hyperbolic paraboloid } \\
\text { If } b=\frac{1}{4}, \text { then it is a parabolic cylinder }
\end{array} \\
& 3 \text { and } 4 z=b(x+y)+g \pm \\
& \sqrt{(b(x+y)+g)^{2}-a(x-y)^{2}-g(x+y)+(1-4 b) x y} \\
& \text { If } b=\frac{1}{2}, a=\frac{1}{4}, g \neq 0 \text {, then it is a couple of non- } \\
& \text { If } b=\frac{1}{2}, a=\frac{1}{4}, g=0 \text {, then it is a double plane } \\
& \text { If } b=\frac{1}{2}, a>\frac{1}{4}, g \neq 0 \text {, then it is an elliptical } \\
& \text { cylinder } \\
& \text { If } b=\frac{1}{2}, a<\frac{1}{4}, g=0 \text {, then it is a couple of non- } \\
& \text { parallel planes } \\
& \text { If } b=\frac{1}{2}, a<\frac{1}{4}, g \neq 0 \text {, then it is a hyperbolic } \\
& \text { cylinder } \\
& \text { If } b \neq \frac{1}{2}, a=b-\frac{1}{4} \text {, then it is a couple of non- } \\
& \text { parallel planes } \\
& \text { If } b<\frac{1}{4}, a \neq b-\frac{1}{4} \text {, then it is a cone } \\
& \text { If } b>\frac{3}{4}, a \neq b-\frac{1}{4} \text {, then it is a cone } \\
& x_{0}=\frac{a \sum_{i=1}^{n} x_{i}^{2}+\frac{2(d-a)}{n-1} \sum_{i, j=1, i>j}^{n} x_{i} x_{j}+c \sum_{i=1}^{n} x_{i}}{d \sum_{i=1}^{n} x_{i}+n c}
\end{aligned}
$$

If $d \neq 0$, then the equation has the form

$$
x_{0}=\frac{a \sum_{i=1}^{n} x_{i}^{2}+\frac{2(1-a)}{n-1} \sum_{i, j=1, i>j}^{n} x_{i} x_{j}+c \sum_{i=1}^{n} x_{i}}{\sum_{i=1}^{n} x_{i}+n c}
$$

If $d=0$, then

$$
x_{0}=a \sum_{i=1}^{n} x_{i}^{2}+\frac{2 a}{1-n} \sum_{i, j=1, i>j}^{n} x_{i} x_{j}+\frac{\sum_{i=1}^{n} x_{i}}{n} .
$$

2. If $a_{0} \neq 0$, imposing symmetry and $0=f(0,0, \ldots, 0)$, we get

$$
x_{0}=\frac{1}{2}\left(-b \sum_{i=1}^{n} x_{i}-c \pm\right.
$$




$$
\sqrt{\left.\left(b \sum_{i=1}^{n} x_{i}+c\right)^{2}-4\left(\sum_{i=1}^{n} x_{i}^{2}+d \sum_{i, j=1, i>j}^{n} x_{i} x_{j}+c \sum_{i=1}^{n} x_{i}\right)\right)} .
$$

From idempotency we obtain

$$
c=-e n
$$

and

$$
1+b n+b^{2} n^{2}=-n-\left(\begin{array}{l}
n \\
2
\end{array}\right) d .
$$

Constraints for the parameters can be obtained in a similar way as for the two variables case.

\section{Concluding remarks}

The quadric hypersurfaces that can be considered as idempotent and symmetric aggregation operators have been studied.

In forthcoming works, other aggregation operators such as t-norms, t-conorms or uninorms that are quadric hypersurfaces will be studied (see [1]),

\section{References}

1. C. Alsina, A, Sklar. (1987) A characterization of continuous associative operations whose graphs are ruled surfaces. Aequationes Mathematicae 33 114-119.

2. G. Beliakov, A. Pradera, T. Calvo. (2008) Aggregation Functions: A Guide for Practitioners. Studies in Fuzziness and Soft Computing.

3. T. Calvo, A. Kolesárova, M. Komorníková, R. Mesiar (2002). Aggregation Operators: Properties, Classes and Construction Methods. In Mesiar, Calvo, Mayor Eds. Aggregation Operators: New Trends and Applications. Studies in Fuzziness and Soft Computing. Springer, 3-104.

4. J. Recasens. (2008) Aggregation Operators and Ruled Surfaces. In T. Alsinet, J. Puyol-Gruart, C. Torras Eds., Artificial Intelligence Research and Development, IOS Press, Amsterdam, 206-214. 